\title{
Search for the QCD Critical Point by Higher Moments of Net-proton Multiplicity Distributions at RHIC *
}

\author{
Xiaofeng Luo (for the STAR Collaboration) \\ Institute of Particle Physics, Central China Normal University, Wuhan 430079, \\ China
}

Key Laboratory of Quark and Lepton Physics (Central China Normal

University), Ministry of Education, Wuhan 430079, China

\begin{abstract}
Higher moments of net-proton multiplicity distributions are applied to search for the QCD critical point. In this paper, we will present measurements for kurtosis $(\kappa)$, skewness $(S)$ and variance $\left(\sigma^{2}\right)$ of net-proton multiplicity distributions at the mid-rapidity $(|y|<0.5)$ and transverse momentum range $0.4<p_{T}<0.8 \mathrm{GeV} / c$ for $\mathrm{Au}+\mathrm{Au}$ collisions at $\sqrt{s_{N N}}$ $=7.7,11.5,39,62.4$ and $200 \mathrm{GeV}, \mathrm{Cu}+\mathrm{Cu}$ collisions at $\sqrt{s_{N N}}=22.4$, 62.4 and $200 \mathrm{GeV}, d+\mathrm{Au}$ collisions at $\sqrt{s_{N N}}=200 \mathrm{GeV}$ and $p+p$ collisions at $\sqrt{s_{N N}}=62.4$ and $200 \mathrm{GeV}$. The moment products $\kappa \sigma^{2}$ and $S \sigma$ of net-proton distributions, which are related to volume independent baryon number susceptibility ratios, are consistent with Lattice QCD and Hadron Resonance Gas (HRG) model calculations at high energies $\left(\sqrt{s_{N N}}=62.4\right.$ and $200 \mathrm{GeV}$ ). Deviations of $\kappa \sigma^{2}$ and $S \sigma$ for the $\mathrm{Au}+\mathrm{Au}$ collisions at low energies $\left(\sqrt{s_{N N}}=7.7,11.5\right.$ and $\left.39 \mathrm{GeV}\right)$ from HRG model calculations are also observed.
\end{abstract}

PACS numbers: 25.75.Ld, 25.75.Dw

\section{Introduction}

The main goal of Beam Energy Scan (BES) program [1] at the Relativistic Heavy Ion Collider (RHIC) is to study the phase structure [2], such as map the QCD phase boundary and search for the QCD critical point [3], of the QCD matter created in heavy ion collision. By tuning the colliding energies of two nuclei from $\sqrt{s_{N N}}=200 \mathrm{GeV}$ to $\sqrt{s_{N N}}=7.7 \mathrm{GeV}$, we can access various region of the QCD phase diagram. Higher moments (variance $\left(\sigma^{2}\right)$,

* Presented at the conference 'Strangeness in Quark Matter 2011', Cracow, Poland, September 18-24, 2011 
skewness $(S)$, kurtosis $(\kappa)$ etc.) of conserved quantities, such as net-baryon, net-charge and net-strangeness, multiplicity distributions are very sensitive to the correlation length [4, 5] and can be directly connected to the corresponding thermodynamic susceptibilities in Lattice QCD [6, 7] and Hadron Resonance Gas (HRG) model [8]. As the volume of the system is hard to determine, the susceptibility ratio, such as $\chi_{B}^{(4)} / \chi_{B}^{(2)}$ and $\chi_{B}^{(3)} / \chi_{B}^{(2)}$, are used to compare with the experimental data as $\kappa \sigma^{2}=\chi_{B}^{(4)} / \chi_{B}^{(2)}$ and $S \sigma=\chi_{B}^{(3)} / \chi_{B}^{(2)}$. Theoretical calculations demonstrate that the experimental measurable netproton (proton number minus anti-proton number) number fluctuations can effectively reflect the fluctuations of the net-baryon number [9]. Higher moments analysis opens a completely new domain and provides quantitative method for probing the bulk properties of the hot dense nuclear matter [2].

\section{Observables}

Experimentally, we measure net-proton number event-by-event wise, $N_{p-\bar{p}}=N_{p}-N_{\bar{p}}$, which is proton number minus antiproton number. In the following, we use $N$ to represent the net-proton number $N_{p-\bar{p}}$ in one event. The average value over whole event ensemble is denoted by $\mu=\langle N\rangle$, where the single angle brackets are used to indicate ensemble average of an event-by-event distributions. The deviation of $N$ from its mean value are defined by

$$
\delta N=N-<N>=N-\mu .
$$

The $r^{\text {th }}$ order central moments are defined as

$$
\mu_{r}=<(\delta N)^{r}>, \mu_{1}=0 .
$$

Then, we can define various order cumulants of event-by-event distributions as

$$
\begin{aligned}
C_{1} & =\mu, C_{2}=\mu_{2}, C_{3}=\mu_{3}, \\
C_{n}(n>3) & =\mu_{n}-\sum_{m=2}^{n-2}\left(\begin{array}{c}
n-1 \\
m-1
\end{array}\right) C_{m} \mu_{n-m} .
\end{aligned}
$$

Once we have the definition of cumulants, various moments can be denoted as

$$
M=C_{1}, \sigma^{2}=C_{2}, S=\frac{C_{3}}{\left(C_{2}\right)^{3 / 2}}, \kappa=\frac{C_{4}}{\left(C_{2}\right)^{2}} .
$$

Then, the moments product $\kappa \sigma^{2}$ and $S \sigma$ can be expressed in term of cumulant ratio

$$
\kappa \sigma^{2}=\frac{C_{4}}{C_{2}}, S \sigma=\frac{C_{3}}{C_{2}}
$$




\section{Background Effects}

Therminator model [10] was applied to study the resonance decay effect, which is a background effect for higher moment analysis, and also to check whether the net-proton fluctuations can reflect the net-baryon number fluctuations. In Fig. 1, we show the event-by-event number distributions of $\mathrm{Au}+\mathrm{Au} 0-5 \%$ most central collisions at $\sqrt{s_{N N}}=200 \mathrm{GeV}$ from Therminator calculations for four cases. Fig. 10 demonstrates that the distribution for the net-proton with resonance decay is wider than the net-proton distributions without decay. By excluding the $\Lambda$ decay nucleon, the net-(proton + neutron $+\Lambda$ ) has narrower distribution than the net(proton+neutron) distributions. Fig. 2 shows centrality dependence of $\kappa \sigma^{2}$ of number distributions. The results for the four cases are consistent with each other within errors, which indicate the effects of resonance decay are small and the net-proton fluctuations can reflect the net-baryon fluctuations. The statistical errors are evaluated by Delta theorem method [11].

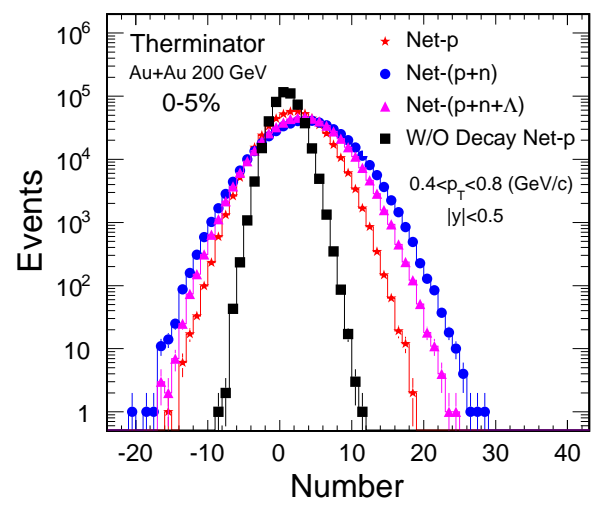

Fig. 1. Event-by-event number distributions for net-proton, net-(proton+neutron), net$($ proton + neutron $+\Lambda)$ and net-proton without resonance decay for $0-5 \%$ most central $\mathrm{Au}+\mathrm{Au}$ collisions at $\sqrt{s_{N N}}=200 \mathrm{GeV}$ from Therminator model.

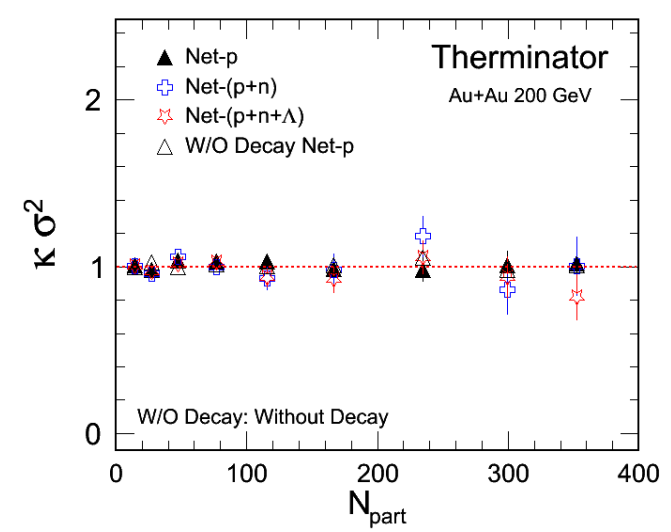

Fig. 2. Centrality dependence of $\kappa \sigma^{2}$ of number distributions of four cases for $\mathrm{Au}+\mathrm{Au}$ collisions at $\sqrt{s_{N N}}=200 \mathrm{GeV}$ from Therminator model.

\section{Experimental Method}

The data presented in this proceedings are obtained using the Solenoidal Tracker at RHIC (STAR). The main subsystem used in this analysis is a large, uniform acceptance cylindrical Time Projection Chamber (TPC) covering a pseudo-rapidity range of $|\eta|<1$ and full azimuthal coverage. To 
ensure the purity and similar efficiency, the protons and anti-protons are identified with the ionization energy loss $(d E / d x)$ measured by the TPC of STAR detector within $0.4<p_{T}<0.8 \mathrm{GeV} / c$ and mid-rapidity $(|y|<0.5)$. Centralities are determined by the uncorrected charged particle multiplicities $\left(d N_{c h} / d \eta\right)$ within pseudo-rapidity $|\eta|<0.5$ measured by the TPC and the centrality bin width correction is used to eliminate volume fluctuations [12. By comparing measured $d N_{c h} / d \eta$ with the Monto Carlo Glauber model results, we can obtain the average number of participant $\left(N_{\text {part }}\right)$ for each centrality.

\section{Results}

In this section, we present beam energy and system size dependence of various moments $(M, \sigma, S, \kappa)$ and moment products $\left(S \sigma\right.$ and $\left.\kappa \sigma^{2}\right)$ of netproton distributions. Those are $\mathrm{Au}+\mathrm{Au}$ collisions at $\sqrt{s_{N N}}=7.7,11.5$, $39,62.4$ (year 2004) and $200 \mathrm{GeV}$ (year 2004), $\mathrm{Cu}+\mathrm{Cu}$ collisions at $\sqrt{s_{N N}}$ $=22.4,62.4,200 \mathrm{GeV}, d+\mathrm{Au}$ at $\sqrt{s_{N N}}=200 \mathrm{GeV}$ (year 2003) and $p+p$ collisions at $\sqrt{s_{N N}}=62.4$ (year 2006), $200 \mathrm{GeV}$ (year 2009). The results for $\mathrm{Au}+\mathrm{Au}$ collisions at $\sqrt{s_{N N}}=62.4$ and $200 \mathrm{GeV}$ have been published in the paper 13. The errors shown in the figures are statistical error only. Centrality dependence of various moments of net-proton distributions for

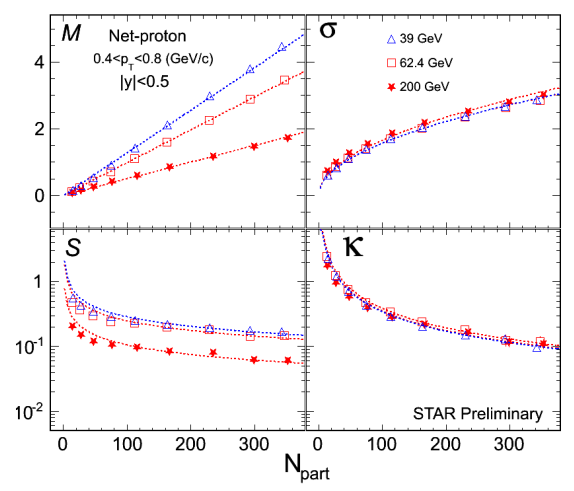

Fig. 3. Centrality dependence of various moments of net-proton multiplicity distributions for $\mathrm{Au}+\mathrm{Au}$ collisions at $\sqrt{s_{N N}}=39,62.4,200 \mathrm{GeV}$. The dashed lines shown in the figure are expectation lines from CLT.

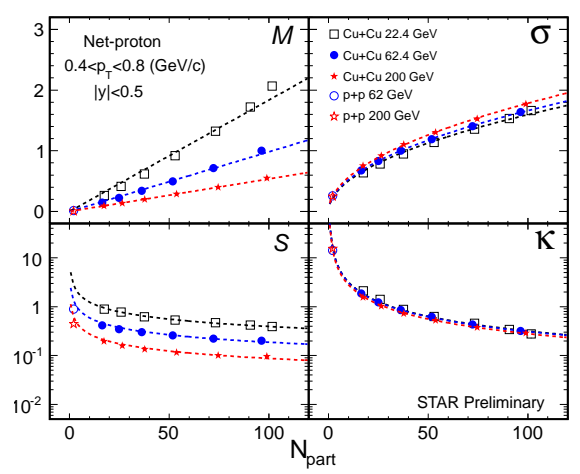

Fig. 4. Centrality dependence of various moments of net-proton distributions for $\mathrm{Cu}+\mathrm{Cu}$ collisions at $\sqrt{s_{N N}}=22.4,62.4$ and $200 \mathrm{GeV}$ and $p+p$ collisions at $\sqrt{s_{N N}}=62.4$ and $200 \mathrm{GeV}$. The dashed lines shown in the figure are expectation lines from CLT.

$\mathrm{Au}+\mathrm{Au}, \mathrm{Cu}+\mathrm{Cu}$ and $p+p$ collisions are shown in Fig. 3 and Fig. 4, respectively. The $M$ and $\sigma$ are found to be monotonically increasing with 
increasing of $N_{\text {part }}$, while the $S$ and $\kappa$ are decreasing. The various moments can be well described by the dashed lines shown in the figures, which are derived from Central Limit Theorem (CLT) by assuming the colliding system consists of many identical and independent emission sources [12, 14]. Energy dependence of $S \sigma$ and $\kappa \sigma^{2}$ for $0-5 \%$ most central $\mathrm{Au}+\mathrm{Au}$ collisions

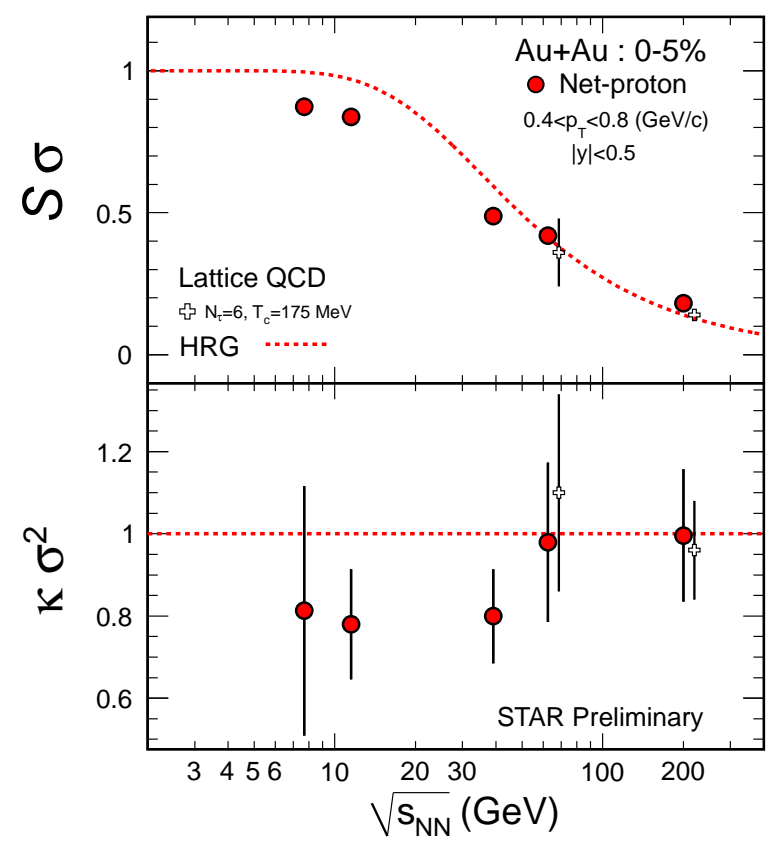

Fig. 5. Energy dependence of moment products $\left(\kappa \sigma^{2}\right.$ and $\left.S \sigma\right)$ of net-proton distributions for $0-5 \%$ most central $\mathrm{Au}+\mathrm{Au}$ collisions. The red dashed lines denote the HRG model calculations, and the empty markers denote Lattice QCD results [2].

are shown in Fig. 5. We find that the data are consistent with Lattice QCD and HRG model calculations at high energies $\left(\sqrt{s_{N N}}=62.4\right.$ and $\left.200 \mathrm{GeV}\right)$, while deviations from HRG model are observed at low energies $\left(\sqrt{s_{N N}}=\right.$ 7.7, 11.5 and $39 \mathrm{GeV}$ ). The possible reasons for the deviations are discussed in [15, 16].

\section{Summary and Outlook}

Higher moments of net-proton distributions are applied to search for the QCD critical point and probe the bulk properties of QCD matters. In 
summary, we present the measurements of higher moments of net-proton distributions for $\mathrm{Au}+\mathrm{Au}, \mathrm{Cu}+\mathrm{Cu}, d+\mathrm{Au}$ and $p+p$ collisions from STAR experiment. The moment products $\kappa \sigma^{2}$ and $S \sigma$ of net-proton distributions from 0-5\% most central $\mathrm{Au}+\mathrm{Au}$ collisions are consistent with Lattice QCD and HRG model calculations at high energies $\left(\sqrt{s_{N N}}=62.4\right.$ and $\left.200 \mathrm{GeV}\right)$, while the results are smaller than HRG model calculations at low energies $\left(\sqrt{s_{N N}}=7.7,11.5,39 \mathrm{GeV}\right)$. The analysis of data from another two energies at $\sqrt{s_{N N}}=19.6$ and $27 \mathrm{GeV}$, which were collected in the year 2011, are ongoing.

\section{Acknowledgement}

The work was supported in part by the National Natural Science Foundation of China under grant No. 11135011.

\section{REFERENCES}

[1] M. M. Aggarwal et al. (STAR Collaboration), arXiv: 1007.2613.

[2] S. Gupta, X. Luo, B. Mohanty, H. G. Ritter, N. Xu, Science 332, 1525 (2011).

[3] R. V. Gavai, S. Gupta, Phys. Rev. D 78, 114503 (2008).

[4] M. A. Stephanov, Phys. Rev. Lett. 102, 032301 (2009).

[5] C. Athanasiou et al., Phys. Rev. D 82 (2010) 074008.

[6] R. V. Gavai and S. Gupta, Phys. Lett. B 696 (2011) 459.

[7] M. Cheng et al., Phys. Rev. D 79, 074505 (2009).

[8] F. Karsch and K. Redlich, Phys. Lett. B 695, 136 (2011).

[9] Y. Hatta and M. A. Stephanov, Phys. Rev. Lett. 91, 102003 (2003).

[10] A. Kisiel et al., Comput. Phys. Commun. 174, 669 (2006).

[11] X. Luo, arXiv: 1109.0593.

[12] X. Luo, J. Phys.: Conf. Ser. 316, 012003 (2011).

[13] M. M. Aggarwal et al. (STAR Collaboration), Phys. Rev. Lett. 105, 022302 (2010).

[14] X. Luo et al., J. Phys. G: Nucl. Phys. 37, 094061 (2010).

[15] M. A. Stephanov, Phys. Rev. Lett. 107, 052301 (2011).

[16] B. Friman et al., Eur. Phys. J. C 71, 1694 (2011). 